\title{
Transient health symptoms of MRI staff working with 1.5 and 3.0 Tesla scanners in the UK
}

\author{
Frank de Vocht • Evridiki Batistatou • Anna Mölter • \\ Hans Kromhout • Kristel Schaap • Martie van Tongeren • \\ Stuart Crozier • Penny Gowland • Stephen Keevil
}

Received: 10 September 2014 / Revised: 12 December 2014 / Accepted: 21 January 2015 / Published online: 13 March 2015

(C) European Society of Radiology 2015

\begin{abstract}
Objectives Recent studies have consistently shown that amongst staff working with MRI, transient symptoms directly attributable to the MRI system including dizziness, nausea, tinnitus, and concentration problems are reported. This study
\end{abstract}

F. de Vocht $(\bowtie)$

School of Social and Community Medicine, University of Bristol, Canynge Hall, 39 Whatley Road, Bristol, UK BS8 2PS

e-mail: frank.devocht@bristol.ac.uk

E. Batistatou

Centre for Occupational and Environmental Health, University of Manchester, Manchester, UK

\section{A. Mölter}

Department of Environmental and Radiological Health Sciences, Colorado State University, Fort Collins, CO, USA

\section{H. Kromhout $\cdot$ K. Schaap}

Institute for Risk Assessment Sciences (IRAS), Utrecht University,

Utrecht, The Netherlands

M. van Tongeren

Centre for Human Exposure Science, Institute of Occupational

Medicine, Edinburgh, UK

S. Crozier

School of Information Technology and Electrical Engineering, University of Queensland, Brisbane, Australia

P. Gowland

Sir Peter Mansfield Magnetic Resonance Centre, University of Nottingham, Nottingham, UK

\section{S. Keevil}

Department of Medical Physics, Guy's and St Thomas' NHS

Foundation Trust, London SE1 7EH, UK

S. Keevil

Department of Biomedical Engineering, King's College London, London SE1 7EH, UK assessed symptom prevalence and incidence in radiographers and other staff working with MRI in healthcare in the UK. Methods One hundred and four volunteer staff from eight sites completed a questionnaire and kept a diary to obtain information on subjective symptoms and work practices, and wore a magnetic field dosimeter during one to three randomly selected working days. Incidence of MRI-related symptoms was obtained for all shifts and prevalence of MRI-related and reference symptoms was associated to explanatory factors using ordinal regression.

Results Incident symptoms related to working with MRI were reported in $4 \%$ of shifts. Prevalence of MRI-related, but not reference symptoms were associated with number of hours per week working with MRI, shift length, and stress, but not with magnetic field strength $(1.5$ and $3 \mathrm{~T})$ or measured magnetic field exposure.

Conclusions Reporting of prevalent symptoms was associated with longer duration of working in MRI departments, but not with measured field strength of exposure. Other factors related to organisation and stress seem to contribute to increased reporting of MRI-related symptoms.

Key Points

- Routine work with MRI is associated with increased reporting of transient symptoms

- No link to the strength of the magnetic field was demonstrated.

- Organisational factors and stress additionally contribute to reporting of MRI-related symptoms

Keywords Epidemiology $\cdot$ Health $\cdot$ Magnetic resonance imaging $\cdot$ Occupational exposure $\cdot$ Magnetic fields

\section{Introduction}

Although MRI has important benefits over other clinical imaging modalities, including that no ionising radiation is used 
for image acquisition, this does not necessarily mean that MRI has no side effects. Acute bioeffects, such as burns, peripheral nerve stimulation (PNS) and safety concerns related to ferromagnetic materials, are reasonably well understood [1], and safety protocols have been put in place to protect patients and staff [2-4]. In the European Union, MRI is within the scope of the Electromagnetic Fields (EMF) Directive, although specifically exempted from the exposure limit values subject to certain conditions and if all proper precautions are in place [5].

However, results from observational studies of MR engineers [6], healthcare and research staff [7], and MR nurses [8] have consistently shown that staff report experiencing various transient symptoms. The frequency of reported complaints was related to the strength of the MRI systems with which staff routinely worked in all studies. Similarly, for patients, unpleasant effects (dizziness and vertigo, for example, but also tachycardia and sweating), some of which are related to the static field, are reported more often during imaging using 7 T systems than with 1.5 T systems [9]. Some of these reported effects may be related to adverse effects on visual perception, spatial orientation, visuomotor performance, working memory, and attention, measured in controlled trials [10-13]. Experimental studies similarly show that exposure to the static magnetic field has a direct negative, but temporary, effect on the vestibular system [14-18]. The effects seem stronger following (head) movements in the heterogeneous stray field, particularly near the outer edge of the magnet bore where the static field changes most rapidly in space, but are not observed with time-varying magnetic fields without the presence of a static gradient magnetic fields [19]. This seems consistent with recent evidence indicating the need for continuing vestibular input from the static field to induce effects [20].

This study aims to describe the pattern of subjective health symptoms self-reported by staff routinely working with MRI in a clinical setting in the UK, and to analyse the impact of magnetic field exposure-related factors as well as other aspects of working conditions on symptom reporting.

\section{Materials and methods}

\section{Study design}

MRI departments at NHS sites in England, Wales, and Scotland were invited to participate through the research team's contacts and an open call at the Institute of Physics and Engineering in Medicine (IPEM) annual conference. When a site agreed and appropriate approvals were obtained, one of the researchers (AM or FV) would visit the site for two to five consecutive days on a random date, organized with each local contact prior to the visit. Staff who worked with MRI on each day of the site visit were asked to participate, with a maximum of three participants per day (limited by available dosimeters).
Ethical approval was obtained from the University of Manchester's Research Ethics Committee (reference number: 12066) and NHS R\&D approval was obtained individually for each of the participating sites.

\section{Data acquisition}

Prior to the start of each participant's first measured shift they were provided with a study information sheet and consent form, and once completed they were given (a) a baseline questionnaire to collect information on occupational history, personal characteristics, medical history, and prevalence of subjective symptoms in the period prior to the measurements; (b) a diary to list all procedures that required access to the MRI suite, details of the MRI systems, and incidence of subjective symptoms during the shift for which they were monitored; and (c) a dosimeter to measure personal exposure to the static and movement-induced, time-varying magnetic fields (SMF and TVMF, respectively) during that particular shift. Participants could be included on multiple days, in which case only the diary and dosimeter were provided on subsequent days. Because questionnaires were completed on the measurement day on site in the company of colleagues, they were not necessarily completed independently.

The baseline questionnaire included questions about transient effects ("subjective symptoms") that have previously (ever) been associated with being in the vicinity of MRI systems [6-8], and although not exclusively caused by exposure to the magnetic fields from MRI they are termed 'MRI-related symptoms' for the purpose of these analyses. In addition, subjective reference symptoms from the Lund Subjective Health Complaint Inventory (SHC) questionnaire [21] experienced within the month prior to measurements were also included to evaluate whether radiographers report certain symptoms more often than, or more severe than the general population; some of which may overlap with the list of MRI-related symptoms (Table 1). The SHC questionnaire is a validated instrument to obtain information about commonly reported subjective health complaints in the general population. It consists of 29 items which are all rated on a 4 -point scale from $0=$ none to $3=$ severe. In addition to the prevalence of these symptoms, their incidence was also recorded in the daily diary for the specific measured shifts.

Stress was measured by five screening questions in the diary, based on selected questions from identified factors of the 'Perceived Stress Questionnaire' (PSQ) [22]. Similar to the PSQ, participants had to select answers from a 4-point scale ranging from 'strongly disagree' to 'strongly agree'. Scores $1-4$ were added up with 20 indicating maximum stress.

We hypothesized that individuals susceptible to effects of magnetic field exposure may be those who are also susceptible to motion sickness. This was specifically investigated based 
Table 1 Overview of health symptoms included in the questionnaires and which, a priori based on previous studies, have been divided into MRI-related and non-MRI related reference symptoms.

\begin{tabular}{ll}
\hline MRI-attributed symptoms & Lund Subjective Health Complaints (SHC) \\
\hline Dizziness/vertigo & Also included in MRI-attributed symptoms \\
Nausea & Headaches \\
Concentration problems & Migraine \\
Memory loss & Tiredness \\
Drowsiness & Dizziness \\
Headaches & Reference symptoms \\
Metallic taste & Cold/flu \\
Illusion of movement & coughing \\
Head ringing & Neck pain \\
Sleep disorder & Upper back pain \\
& Lower back pain \\
& Anxiety \\
& Extra heart beats \\
& Heat flushes \\
& Stomach discomfort \\
& Heartburn \\
& Breathing difficulties \\
& Allergies \\
& Eczema \\
& Chest pain \\
& \\
& \\
& \\
& \\
& \\
& \\
& \\
&
\end{tabular}

on the MSSQ-Short questionnaire [23]. Motion sickness susceptibility was separately calculated for susceptibility during childhood, during adulthood, and combined, based on the methodology outlined in [23].

\section{Personal exposure measurements}

Personal shift-exposure to the SMF and motion-induced TVMF from the MRI scanner was measured in real-time using a personal dosimeter (Magnetic Field Dosimeter, University of Queensland, Australia) worn by each participant at the hip. Measurements in all three orthogonal directions were collected at a sampling rate of $50 \mathrm{~Hz}$. Total exposure to the SMF (B) was obtained in millitesla $(\mathrm{mT})$ and total exposure to motioninduced TVMF $(\mathrm{dB} / \mathrm{dt})$ in $\mathrm{mT} / \mathrm{s}$. More information about the dosimeter can be found in [24-26]. Participants could be measured on multiple days $(1-3)$, and in this case, exposure was averaged across shifts as a proxy for average, typical exposure.

\section{Statistical analyses}

Reported health complaints were grouped into 'MRI-related' and 'non-MRI-related' based on similar classifications in previous studies [6-8], and the total number of complaints reported by each participant was used as a measure of severity. Associations between the prevalence of health complaints and various exposure-related and organisational determinants were analysed using univariate and multivariate ordinal logistic regression using the MASS package in $R$ (version 3.0.1) [27]. Because the majority of participants were radiographers the analyses were conducted for the whole, exposed population, but also for the radiographers separately. Effect sizes are expressed in terms of the increased odds of reporting an additional symptom with one unit increase in the explanatory variable. Ninety-five percent confidence intervals (CI) were calculated based on profile likelihood, and these are presented instead of $p$-values.

\section{Results}

An overview of the study population is shown in Table 2. A total of 117 people from eight NHS sites across the UK participated in the study. One hundred and four of these routinely worked with MRI and 13 were controls (not included in further analyses). Of these, $70 \%$ were women and their average age was 40 years. They had worked on average for 8 years with MRI (range 1 to 23 years). The majority of participants $(71 \%)$ were radiographers and primarily $(81 \%)$ worked with 1.5 T MRI systems. Measured shift-average SMF exposure was about $30 \mathrm{mT}$, and motion-induced TVMF about $54 \mathrm{mT} / \mathrm{s}$. Histograms of the measured exposure distributions are shown in Fig. 1 and are all approximately log-normally distributed. $53 \%$ of participants reported at least one symptom which they specifically attributed to their work with MRI, and $74 \%$ reported experiencing at least one of the reference SHC symptoms in the previous month.

MRI-related symptoms were reported during $4 \%$ of the measured shifts, and were more specifically 'slight dizziness after cleaning bore', 'dizzy feeling for $30 \mathrm{~s}$ ', 'slightly dizzy, but busy', 'slight headache', 'very mild headache', and 'eye strain/dizzy'. Magneto-phosphenes were not reported. Two participants reported experiencing musculoskeletal complaints during patient transfer, but these were not included in the incidence rate, while one participant reported eye strain and dizziness during three different procedures on the same day. MRI-related symptoms were reported during cleaning, patient positioning, and injection of contrast agent, but because of the low incidence, no further statistical analyses were done on these data.

The SHC questionnaire was previously administered to the general population in Norway [21], but to our knowledge not in the UK. Therefore, reporting by MRI staff was compared to published data from the Norwegian population (Table 3). Consistent with an anticipated healthy worker effect [28], the frequency and severity of reported reference, non-MRI related symptoms was generally lower in participating MRI- 
Table 2 Overview of study population

\begin{tabular}{|c|c|}
\hline \multicolumn{2}{|l|}{ Characteristic } \\
\hline Number of Sites & 8 \\
\hline Number of Participants & 117 \\
\hline Number of Measured days & 166 \\
\hline Sex (\% females) & $70 \%$ \\
\hline Age in years ${ }^{2}$ (mean, range) & $40(23-65)$ \\
\hline Unexposed (controls) & 13 \\
\hline Exposed & 104 \\
\hline \multicolumn{2}{|l|}{ Job title ${ }^{1}$} \\
\hline Radiographers & 74 \\
\hline Radiologists & 3 \\
\hline Anaesthetists & 5 \\
\hline Nurses/assistants & 19 \\
\hline Other (ODP, fellow) & 3 \\
\hline \multicolumn{2}{|l|}{ MRI systems } \\
\hline $1.5 \mathrm{~T}$ & 87 \\
\hline $1.5+3.0 \mathrm{~T}$ & 17 \\
\hline Average total years working with MRI & $8(<1-23)$ \\
\hline Average $\mathrm{h} /$ week working with MRI & $27(0.5-40)$ \\
\hline Average B exposure (range) & $29.8 \mathrm{mT}(5.7-720.3)$ \\
\hline Average dB/dt exposure (range) & $54.4 \mathrm{mT} / \mathrm{s}(8.9-2,618.7)$ \\
\hline \multicolumn{2}{|l|}{$\begin{array}{l}\text { MRI-related symptoms during or immediately } \\
\text { after working with } \mathrm{MRI}^{3}\end{array}$} \\
\hline 0 & $47 \%$ \\
\hline $1-2$ & $42 \%$ \\
\hline $3-4$ & $9 \%$ \\
\hline $5+$ & $2 \%$ \\
\hline \multicolumn{2}{|c|}{ Non-MRI related complaints in the previous month ${ }^{3}$} \\
\hline 0 & $26 \%$ \\
\hline $1-2$ & $29 \%$ \\
\hline $3-4$ & $23 \%$ \\
\hline $5+$ & $22 \%$ \\
\hline $\begin{array}{l}\text { Incidence of MRI-related symptom reported } \\
\text { during } 166 \text { shifts }\end{array}$ & $4 \%$ \\
\hline
\end{tabular}

\begin{tabular}{l}
\hline job titles collapsed to generic categories; ${ }^{2}$ not reported by six partici- \\
pants; ${ }^{3} 10$ single MRI-related complaints and 14 non-MRI related \\
complaints
\end{tabular}

workers than in the general population. Statistically significant differences were observed for cold/flu, neck pain, heartburn, allergies, eczema, and chest pain. A similar pattern compared to the general population was observed for frequency of reports, but not for severity for MRI-related symptoms. Symptoms with a higher severity score were more often reported by the participants than they were reported in the (Norwegian) general population; the differences, however, were not statistically significant.

Results of ordinal logistic regression, in which the symptoms ever experienced and self-attributed to work with MRI, were analysed and are shown in Table 4. Age had a small, negative effect on reporting for both MRI-related and nonMRI related subjective health symptoms. Radiographers had an eightfold increased risk of experiencing MRI-attributed symptoms $(\mathrm{OR}=8.05,95 \%$ confidence interval 1.96 to 54.84), and nurses and assistants reported a two- to threefold increased risk, although not statistically significant (95\% CI $0.44-18.71)$, compared to clinicians. Interestingly, radiographers also reported more reference symptoms a priori not related to their work with MRI, although the risk was much lower $(\mathrm{OR}=2.61)$ and the difference not statistically significant. The number of self-reported MRI-related symptoms was further associated with average hours per week working with MRI (OR=1.06, $95 \%$ CI 1.03 to 1.07$)$, and the number of times the participant went in the MRI suite during measured shifts $(\mathrm{OR}=1.03,95 \% \mathrm{CI} 1.00-1.07)$, the average shift duration $(\mathrm{OR}=1.34,1.10-1.67)$ and selfperceived stress during the measured days $(\mathrm{OR}=1.14,95 \%$ CI $1.00-1.29$ ). Adult, but not childhood, susceptibility to motion sickness was associated with increased reporting $(\mathrm{OR}=1.07,95 \% \mathrm{CI} 1.01-1.13)$. The univariate results for just the radiographers were similar to those for all participants (data not shown).

In multivariate analyses, all variables that were significant in the univariate analysis remained significant. Furthermore, job title, although an important predictor by itself was not included in the multivariate analysis because it should be regarded as a proxy for other factors. After adjustment for confounding factors (age, stress, and motion sickness susceptibility during adulthood), neither the strength of the MRI system, nor measured average or peak exposure to the SMF or TVMF were associated with increased reporting of symptoms. Instead, average shift duration was a predictor of MRIrelated, but not reference symptoms (OR $=5.64,95 \%$ CI 1.87 - 18.78). The average hours of working with MRI in a week was also related to increased risk of reporting additional MRIrelated symptoms, but not reference symptoms (ORs 1.51, $95 \%$ CI $1.16-2.03$, and 1.01, $95 \%$ CI $0.98-1.04$, respectively). Shift duration and hours per week working with MRI were only moderately correlated ( $\mathrm{r} \sim 0.4)$, but their statistically significant interaction (OR=0.95 95\% CI 0.92 - 0.99) suggests that risk does not linearly increase with increased shift duration and hours per week working with MRI, but that that the risk function is supralinear. The results for radiographers alone are similar to those of all MRI staff in the study. Additional sensitivity analyses in which highest exposures were removed (outliers) yielded similar results as presented in Table 4.

Reporting of MRI-related symptoms was also independently associated with perceived stress. Given the type of symptoms this does make sense, but surprisingly this is not observed for the reference symptoms $(\mathrm{OR}=0.97,95 \% \mathrm{CI} 0.86$ $-1.10)$, leaving open the question whether there is some other factor correlated to working with MRI, but unobserved in this 
Fig. 1 histogram of measured shift weighted-average (SWA) and peak exposure to static and time-varying magnetic fields
SWA static field (B)

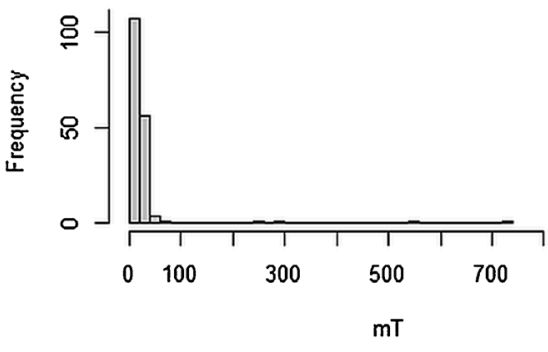

Peak static field (B)

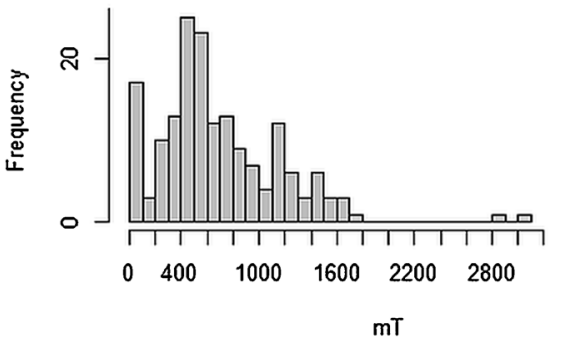

SWA time-varying field $(d B / d t)$

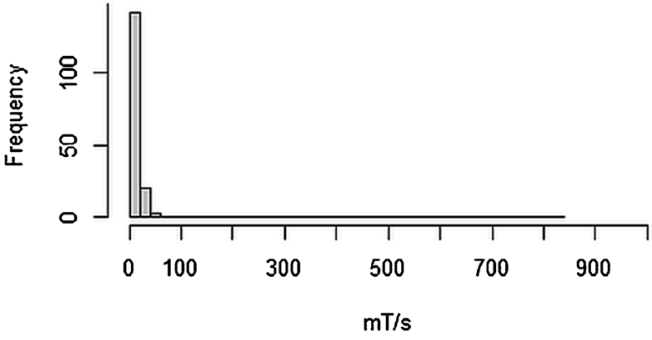

Peak time-varying field (dB/dt)

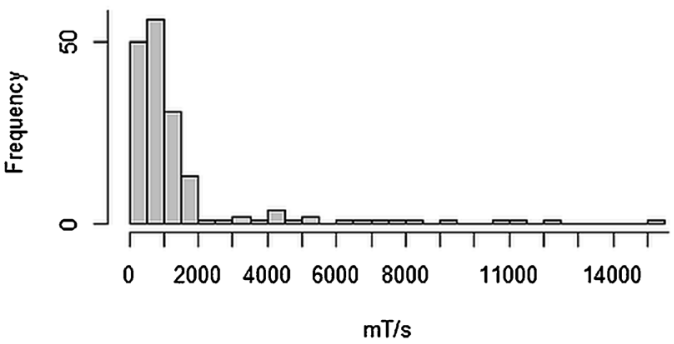

Table 3 Comparison of self-reported complaints from the Lund Subjective Health Complaints (SHC) questionnaire between the study population and Norwegian lay population, stratified by whether complaints have also been linked to MRI

\begin{tabular}{|c|c|c|c|c|c|c|}
\hline & \multicolumn{3}{|c|}{ Any $($ score $>0)(\%)$} & \multicolumn{3}{|c|}{ Severe $($ score $=3)(\%)$} \\
\hline & $\begin{array}{l}\text { MRI workers } \\
\text { \%any }\end{array}$ & $\begin{array}{l}\text { General population* } \\
\text { \%any }\end{array}$ & $p$-value** & $\begin{array}{l}\text { MRI workers } \\
\text { \%severe }\end{array}$ & $\begin{array}{l}\text { General population* } \\
\text { \%severe }\end{array}$ & $p$-value** \\
\hline \multicolumn{7}{|c|}{ Health complaint also included in MRI-related complaints } \\
\hline Headache & 45.2 & 55.4 & 0.05 & 2.9 & 2.1 & 0.76 \\
\hline Migraine & 9.6 & 8.2 & 0.66 & 1.9 & 1.1 & 0.66 \\
\hline Tiredness & 58.7 & 61.3 & 0.60 & 3.8 & 2.8 & 1.00 \\
\hline Dizziness & 11.5 & 17.0 & 0.17 & 1.0 & 0.4 & 0.48 \\
\hline \multicolumn{7}{|c|}{ Other subjective health complaints } \\
\hline Cold or flu & 31.7 & 43.4 & 0.02 & 1.0 & 3.2 & 0.12 \\
\hline Coughing & 22.1 & 20.4 & 0.70 & 1.9 & 1.7 & 1.00 \\
\hline Neck pain & 25.0 & 45.0 & 0.00 & 2.9 & 2.5 & 1.00 \\
\hline Upper back pain & 20.2 & 25.8 & 0.24 & 1.0 & 2.0 & 0.50 \\
\hline Lower back pain & 37.5 & 47.8 & 0.05 & 2.9 & 3.6 & 0.62 \\
\hline Anxiety & 15.4 & 10.9 & 0.19 & 0.0 & 0.6 & 1.00 \\
\hline Extra heartbeats & 6.7 & 10.7 & 0.24 & 0.0 & 0.1 & 1.00 \\
\hline Heat flushes & 10.6 & 13.0 & 0.54 & 1.0 & 0.5 & 0.53 \\
\hline Stomach discomfort & 11.5 & 12.3 & 1.00 & 0.0 & 0.6 & 1.00 \\
\hline Heartburn & 8.7 & 18.1 & 0.01 & 0.0 & 0.4 & 1.00 \\
\hline Breathing difficulties & 4.8 & 6.9 & 0.54 & 1.0 & 0.4 & 0.48 \\
\hline Allergies & 3.8 & 15.4 & 0.00 & 0.0 & 1.4 & 0.24 \\
\hline Eczema & 3.8 & 14.6 & 0.00 & 1.0 & 1.1 & 1.00 \\
\hline Chest pain & 1.9 & 7.9 & 0.02 & 0.0 & 0.5 & 1.00 \\
\hline
\end{tabular}

Bold indicates statistically significant $(\mathrm{p}<0.05)$ differences between MRI workers and the general population

* For the Norwegian population; data obtained from [21]. Sex-weighted to match population of this study

** Fisher's Exact test, calculated based on counts obtained from [21] 


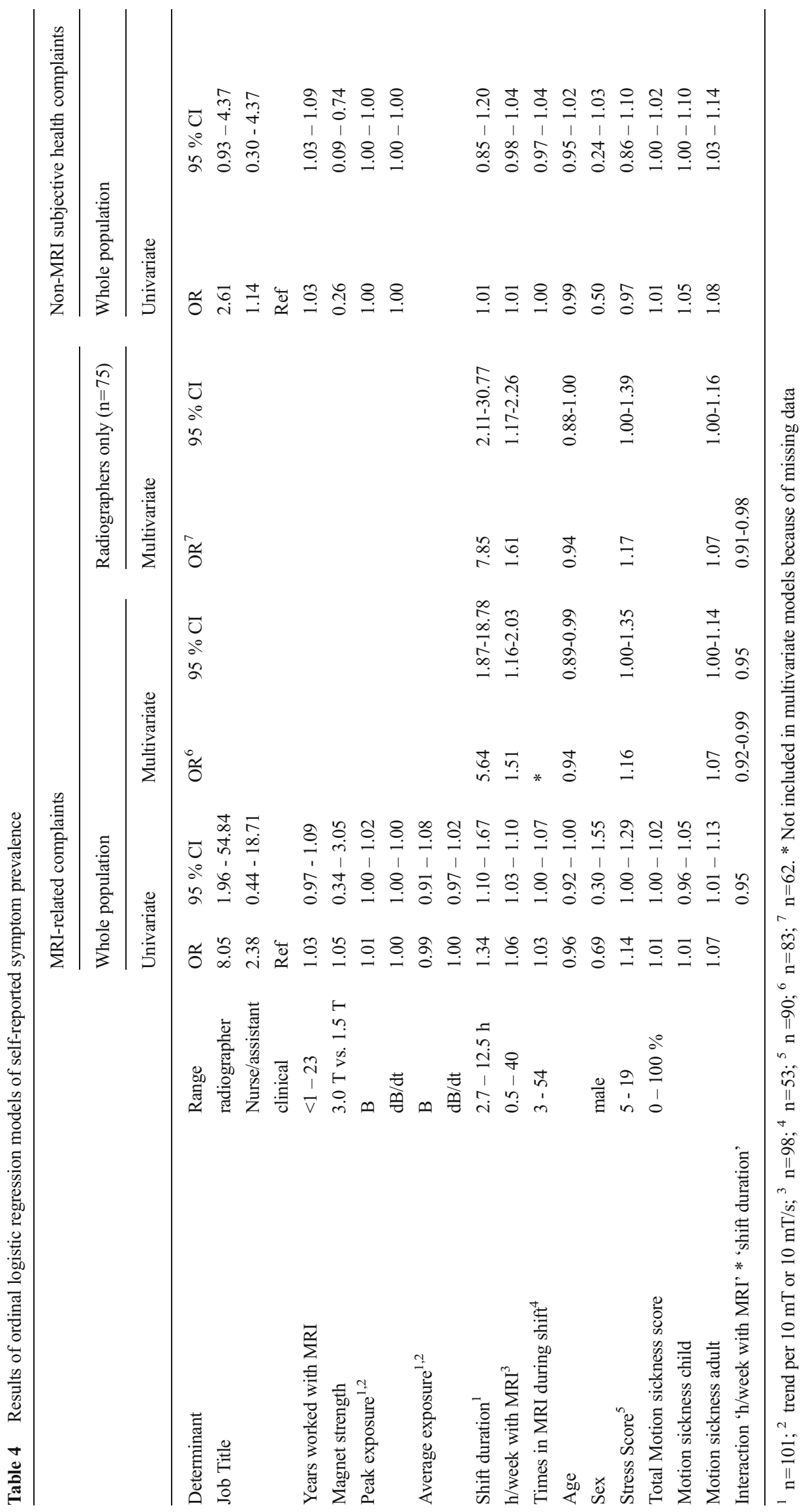


study that could explain this association. Furthermore, although motion sickness susceptibility during adulthood was associated with increased reporting of MRI-related symptoms, a similar increased risk was observed for the reference symptoms suggesting that this is likely caused by some other factor not directly related to exposure to the magnetic fields.

\section{Discussion}

Compared to the general population, staff working with MRI reported subjective health symptoms less often, which is consistent with the expected healthy worker effect observed in many studies comparing the occupationally active with the general population [28]. However, MRI workers reported more symptoms of the set a priori assigned as MRI-related, which may point to a contribution from their work with MRI systems. Although a relatively small study, the results indicated that $53 \%$ of people routinely working with MRI in NHS hospitals have experienced at least one subjective health complaint selfattributed to MRI. This is comparable to the $47 \%$ reported in a study amongst MRI nurses in Sweden [8]. However, this may not necessarily be different from a comparable population, since neither study included a control population. A Dutch study amongst MR and X-ray system engineers indicated that the incidence of reported symptoms was about twice as high in the MR department (32\%) compared to the X-ray department (15\%) [6], which may point to a contribution of magnetic field exposure from the MRI systems. In our study, the reported incidence of self-reported symptoms during the measured shifts (other than musculoskeletal) was much lower (4\%), which can be explained by differences in work between healthcare staff and MR manufacturing engineers. The incidence is also somewhat lower than the 5-9\% observed for a selected set of 'SMF core symptoms' reported for 1.5 and $3 \mathrm{~T}$ systems in a study on clinical and research facilities in the Netherlands, but much lower than the $28-35 \%$ reported for a larger set of 'SMF target symptoms' in the same population [7]. The control population in that study reported at least one SMF core and/or SMF target symptom in $1 \%$ and $25 \%$ of shifts, respectively.

We did not observe an association of increased prevalence of symptoms with increasing exposure, which puts these results at odds with previous studies [6-8] and with experimental work $[11,12,29,30]$. Possibly this may be ascribed to the fact that the variation in exposure in our study was not large enough; we only had staff working with $1.5 \mathrm{~T}$ or $1.5 \mathrm{~T}$ and $3 \mathrm{~T}$ systems, while other studies included exposures ranging from 0 (non-exposed controls) up to $7 \mathrm{~T}$. However, reporting of the reference symptoms decreased with increased magnet strength, which may suggest some residual confounding we could not correct for. Also, information on consumption of coffee and alcohol were not collected because in previous studies $[6,13,19]$ these did not appear to be important factors, while they are also unlikely to be confounders in this context. Information on menstrual cycle, which may be related to symptom reporting, but is similarly also unlikely to be related to exposure, was also not collected.

Instead, we observed an association of increased reporting of MRI-related symptoms with shift duration and average hours per week working with MRI, which may indicate that duration of working in the vicinity of MRI systems (and thereby exposure to MRI-generated magnetic fields) is associated with increased reporting of symptoms. Alternatively, since no associations with shift-average exposures were observed it could also indicate that longer shifts, regardless of MF exposure are associated with increased risk of experiencing symptoms. The former possibility is supported by the fact that similar associations are not observed for the reference symptoms, while the latter may be in line with the observed association between perceived stress and MRI-related symptoms. The number of hours working with MRI per week and the shift length were only moderately correlated with the number of times participants went into the MRI suite $(\mathrm{r} \sim 0.31$ and 0.60 , respectively) and these variables were not correlated to shiftaverage SMF exposure ( $\mathrm{r} \sim 0.03)$ or TVMF ( $\mathrm{r} \sim 0.02)$, indicating that shift-average magnetic field exposure plays a minor role in the observed associations. It has been shown previously that stress itself is not caused by exposure to the SMF from MRI systems directly [31], so this would be due to organisational and managerial issues instead, likely related to shift duration. However, it is important to realize that although it is generally assumed that if anything, the intensity of exposure to the magnetic field ( $\mathrm{B}$ or $\mathrm{dB} / \mathrm{dt}$ ) is related to symptoms, for many of the self-reported symptoms in this study the mechanisms by which these would occur remains unknown.

Our results further indicated, although not statistically significant, that women report MRI-related symptoms more often than men, which is in line with previous results [29]. Our results also indicated that motion sickness susceptibility is not likely to be a useful predictor of magnetic field susceptibility because a similar association was observed for reference symptoms, which is surprising given that experimental studies have shown that exposure to the SMF directly affects the vestibular system in humans [17, 20,32] and animals [33].

Our study had several limitations. This was a relatively small study of only 104 participants which had implications for statistical power. This also prohibited more sophisticated analyses such as investigation of clustering of symptoms within sites using multi-level models [34] as a result of questionnaires not always being completed independently.

Furthermore, because of the study design, which focussed on measuring exposure patterns, we also did not have a control group such as CT radiographers.

Also, it is important to emphasize that exposure was measured after prevalence of symptoms was registered. We used measured exposure during these shifts as an indication of average exposure, but this may have not been a good proxy for average exposure 
over a longer period. A measurement survey in the Netherlands showed large between-shift variability in exposure for radiographers and other clinical staff [26]. Measured exposure was intended to be related to symptom incidence reported in the diaries, but these were not frequent enough ( $4 \%$ of shifts) for meaningful statistical analysis. Furthermore, we looked at measured average and peak exposure only, but these may not be the most relevant exposure metrics (for example, based on thresholds or maximum levels). Further work is required to establish which metrics should be measured in future studies.

And finally, although we compared the prevalence of MRIrelated symptoms to those previously reported, our set of selected 'MRI-related' symptoms is not directly comparable to [7], and falls somewhere in between their core and target symptoms, which is an important contribution to observed differences in reported prevalence.

Nonetheless, this is one of only a few studies investigating the prevalence and incidence of acute and transient symptoms in radiographers and other healthcare workers routinely working with MRI systems. These results support previous findings that routine work with MRI is associated with reporting of a specific set of symptoms and that these occur in roughly $4 \%$ of shifts with $1.5-3 \mathrm{~T}$ systems. Our results further suggest that in our survey, reporting of these specific symptoms is primarily related to duration of work with MRI systems and not to the strength of the systems or the shift-average magnetic field exposure, indicating that shift duration and perceived stress not related to the MRI system were important contributing factors.

Acknowledgments The scientific guarantor of this publication is Dr. Frank de Vocht. The authors of this manuscript declare relationships with the following companies: SK developed, produces, and licenses the dosimeters used in this study through a University of Queensland company. This study has received funding by The COLT Foundation. More than one of the authors has significant statistical expertise. Institutional review board approval was obtained. Written informed consent was obtained from all subjects (patients) in this study. No study subjects or cohorts have been previously reported. Methodology: prospective and retrospective, cross-sectional study/observational, multicenter study.

The authors would like to thank all participants who volunteered for this study, as well as all local coordinators.

Funding source The study was funded by a research grant from The COLT Foundation (grant number $\mathrm{CF} / 01 / 11$ ).

\section{References}

1. Shellock F (2001) Magnetic resonance procedures: health effects and safety. CRC Press, Boca Raton

2. ICNIRP (2009) Guidelines on limits of exposure to static magnetic fields. Health Phys 96:10

3. ICNIRP (2009) Amendment to the ICNIRP "Statement on medical Magnetic Resonance (MR) procedures: protection of patients". Health Phys 97:3
4. ICNIRP (2014) Guidelines for limiting exposure to electric fields induced by movement of the human body in a static magnetic field and by time-varying magnetic fields below $1 \mathrm{~Hz}$. Health Phys 106:8

5. Parliament E (2013) Directive 2013/35/EU of the European Parliament and of the council of 26 june 2013 on the minimum health and safety requirements regarding the exposure of workers to the risks arising from physical agents (electromagnetic fields) (20th individual Directive within the meaning of Article 16(1) of Directive 89/391/EEC) and repealing Directive 2004/40/EC. Official Journal of the European Union L179/1

6. de Vocht F, van Drooge H, Engels H, Kromhout H (2006) Exposure, health complaints and cognitive performance among employees of an MRI scanners manufacturing department. J Magn Reson Imaging 23: 197-204

7. Schaap K, Christopher-de Vries Y, Mason CK, de Vocht F, Portengen L, Kromhout H (2014) Occupational exposure of healthcare and research staff to static magnetic stray fields from 1.5-7 Tesla MRI scanners is associated with reporting of transient symptoms. Occup Environ Med 71:423-429

8. Wilen J, de Vocht F (2011) Health complaints among nurses working near MRI scanners-a descriptive pilot study. Eur J Radiol 80:510 513

9. Heilmaier C, Theysohn JM, Maderwald S, Kraff O, Ladd ME, Ladd SC (2011) A large-scale study on subjective perception of discomfort during 7 and 1.5 T MRI examinations. Bioelectromagnetics 32:610 619

10. van Nierop LE, Slottje P, van Zandvoort MJ, de Vocht F, Kromhout H (2012) Effects of magnetic stray fields from a 7 tesla MRI scanner on neurocognition: a double-blind randomised crossover study. Occup Environ Med 69:759-766

11. de Vocht F, Stevens T, van Wendel-de-Joode B, Engels H, Kromhout $\mathrm{H}$ (2006) Acute neurobehavioral effects of exposure to static magnetic fields: analyses of exposure-response relations. J Magn Reson Imaging 23:291-297

12. de Vocht F, Glover P, Engels H, Kromhout H (2007) Pooled analyses of effects on visual and visuomotor performance from exposure to magnetic stray fields from MRI scanners: application of the Bayesian framework. J Magn Reson Imaging 26:1255-1260

13. de Vocht F, van-Wendel-de-Joode B, Engels H, Kromhout H (2003) Neurobehavioral effects among subjects exposed to high static and gradient magnetic fields from a 1.5 Tesla magnetic resonance imaging system-a case-crossover pilot study. Magn Reson Med 50:670 674

14. Antunes A, Glover PM, Li Y, Mian OS, Day BL (2012) Magnetic field effects on the vestibular system: calculation of the pressure on the cupula due to ionic current-induced Lorentz force. Phys Med Biol 57:4477-4487

15. Glover PM, Cavin I, Qian W, Bowtell R, Gowland PA (2007) Magnetic-field-induced vertigo: a theoretical and experimental investigation. Bioelectromagnetics 28:349-361

16. Roberts DC, Marcelli V, Gillen JS, Carey JP, Della Santina CC, Zee DS (2011) MRI magnetic field stimulates rotational sensors of the brain. Curr Biol 21:1635-1640

17. Theysohn JM, Kraff O, Eilers K et al (2014) Vestibular effects of a 7 Tesla MRI examination compared to $1.5 \mathrm{~T}$ and $0 \mathrm{~T}$ in healthy volunteers. PLoS One 9:e92104

18. van Nierop LE, Slottje P, Kingma H, Kromhout H (2013) MRIrelated static magnetic stray fields and postural body sway: a double-blind randomized crossover study. Magn Reson Med 70: 232-240

19. de Vocht F, Liket L, De Vocht A et al (2007) Exposure to alternating electromagnetic fields and effects on the visual and visuomotor systems. Br J Radiol 80:822-828

20. Mian OS, Li Y, Antunes A, Glover PM, Day BL (2013) On the vertigo due to static magnetic fields. PLoS One 8:e78748 
21. Eriksen HR, Ihlebaek C, Ursin H (1999) A scoring system for subjective health complaints (SHC). Scand J Public Health 27:63-72

22. Levenstein S, Prantera C, Varvo V et al (1993) Development of the Perceived Stress Questionnaire: a new tool for psychosomatic research. J Psychosom Res 37:19-32

23. Golding JF (2006) Predicting individual differences in motion sickness susceptibility by questionnaire. Personal Individ Differ 41:9

24. de Vocht F, Muller F, Engels H, Kromhout H (2009) Personal exposure to static and time-varying magnetic fields during MRI system test procedures. J Magn Reson Imaging 30:1223-1228

25. Fuentes MA, Trakic A, Wilson SJ, Crozier S (2008) Analysis and measurements of magnetic field exposures for healthcare workers in selected MR environments. IEEE Trans Biomed Eng 55:1355-1364

26. Schaap K, Christopher-de Vries Y, Crozier S, De Vocht F, Kromhout $\mathrm{H}$ (2014) Exposure to static and time-varying magnetic fields from working in the static magnetic stray fields of MRI scanners: a comprehensive survey in the Netherlands. Ann Occup Hyg. doi:10.1093/ annhyg/meu057

27. Team RDC (2008) R: a language and environment for statistical computing. R Foundation for Statistical Computing, Vienna
28. Li CY, Sung FC (1999) A review of the healthy worker effect in occupational epidemiology. Occup Med (Lond) 49:225-229

29. Heinrich A, Szostek A, Meyer P et al (2014) Women are more strongly affected by dizziness in static magnetic fields of magnetic resonance imaging scanners. Neuroreport. doi:10.1097/WNR. 0000000000000225

30. Heinrich A, Szostek A, Meyer P et al (2013) Cognition and sensation in very high static magnetic fields: a randomized case-crossover study with different field strengths. Radiology 266:236-245

31. Gilles M, Paslakis G, Heinrich A et al (2013) A cross-over study of effects on the hypothalamus-pituitary-adrenal (HPA) axis and the sympathoadrenergic system in magnetic field strength exposure from 0 to 7 T. Stress $16: 172-180$

32. Glover PM, Li Y, Antunes A, Mian OS, Day BL (2014) A dynamic model of the eye nystagmus response to high magnetic fields. Phys Med Biol 59:631-645

33. Ward BK, Tan GX, Roberts DC, Della Santina CC, Zee DS, Carey JP (2014) Strong static magnetic fields elicit swimming behaviors consistent with direct vestibular stimulation in adult zebrafish. PLoS One 9:e92109

34. Snijders T, Bosker R (1999) Multilevel analysis. SAGE Publications Ltd, London 